\title{
New guide for first order multiplet analysis by modified $J$ doubling in the frequency domain
}

\author{
Federico del Río-Portilla, ${ }^{\mathrm{a}}{ }^{\mathrm{E}}$ Ernesto Sánchez-Mendoza, ${ }^{\mathrm{a}}$ Víctor U. Constantino-Castillo, \\ and J. Antonio del Río \\ a Instituto de Química, Universidad Nacional Autónoma de México, Ciudad Universitaria s/n, \\ México, D. F. 04510, México ${ }^{b}$ Centro de Investigación en Energía, Universidad Nacional \\ Autónoma de México, Temixco, Morelos, México \\ E-mail: jfrp@servidor.unam.mx
}

(received 17 Sep 03; accepted 22 Jan 03; published on the web 25 Jan 04)

\begin{abstract}
Pattern recognition is extremely important for both structure elucidation and stereochemical studies as a means for the measurement of spin-spin coupling constants. Many treatises deal with the formation of splitting trees to create a pattern for a given set of spin-spin coupling constants. We present here a general systematic procedure to easily analyze first order multiplets in NMR spectroscopy. This method has the advantage of measuring coupling constants while deconvolving the signals at the same time using an extension of the modified $J$ doubling in the frequency domain.
\end{abstract}

Keywords: $J$ Doubling, spin-spin coupling constant, deconvolution, convolution, pattern recognition

\section{Introduction}

The analysis of complex multiplets is an important asset for chemists. An awareness of the number of couplings present in a specific multiplet and their values is essential for proper NMR spectra interpretation. These data let us determine the number of neighbors interacting with a nucleus. Nowadays, high field NMR magnets have become common devices in most NMR laboratories. This new equipment allows many NMR signals to become first order and many overlapped ones to be resolved. However, the interpretation and extraction of new-resolved signals is not an easy task. In this paper, we present an easy procedure to understand the pattern of complex NMR multiplets and, at the same time, accurately determine the value of spin-spin coupling constants. 
The interpretation of NMR signals presents a real challenge for chemists. Nevertheless, few papers have been written on pattern recognition, ${ }^{1-3}$ so there is no easy way of explaining those reported methods. On the other hand, many papers have been written on how to accurately measure coupling constants ${ }^{4-11}$ in simplex and in complex multiplets. ${ }^{12-13}$ We propose a new procedure based on the modified $J$ doubling method. ${ }^{13}$ According to our experience, this method is one of the most accurate, versatile and fast techniques to measure and to deconvolve NMR multiplets. As a requirement for a better understanding of the method, we need to remember both convolution and deconvolution mathematical concepts; so, we proceed to explain them in the next section. Also, in order to write a self-contained paper, we describe the modified J doubling method in the second section. Our main proposal, simultaneous deconvolution of equal couplings constants, is presented in the third section. The results on simulated and real NMR spectra appear in the Results and Discussion section. We close the paper with a section explaining the experimental setup.

\section{Convolution and deconvolution}

Tree diagrams easily explain NMR multiplets. This scheme is a simplification of the mathematical process known as convolution, ${ }^{9,13}$ Figure 1. For instance, a doublet can be obtained from the convolution (represented by the symbol $\otimes$ ) of a singlet with a pair of delta functions, ${ }^{14}$ which could be separated by $J_{x}$, Figure 1a, producing a doublet with a splitting of $J_{x}$, Figure 1b, as in the tree diagram. If this doublet is convoluted again with a pair of delta functions separated by $J_{y}$, Figure 1c, the result will be a double of doublets defined by $J_{x}>J_{y}$, Figure $1 \mathrm{~d}$, having the same result as in the tree diagram.

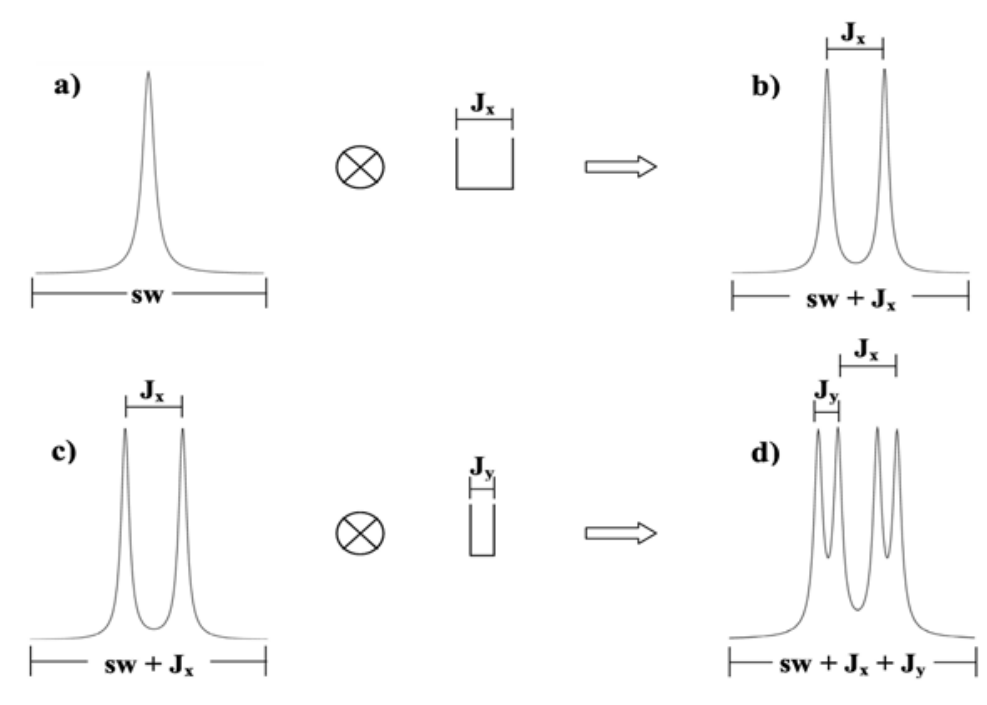

Figure 1. Convolution process of a singlet and a doublet to generate a doublet and a double of doublets. 
The inverse process, going from the double of doublets to a singlet, is known as deconvolution, which is indeed the interpretation done by chemists. The coupling constant values are a requirement to perform the mathematical procedure, as in pattern recognition. $\mathrm{J}$ doubling in the frequency domain is a simple and easy method for measuring coupling constants needed for the deconvolution. ${ }^{15}$ Its modification was developed to deconvolve NMR signals in a very simple manner. Measurements obtained by $\mathrm{J}$ doubling and modified $\mathrm{J}$ doubling are identical. These two characteristics make of the modified $\mathrm{J}$ doubling a very useful technique. We proceed to explain how the modified J doubling method works.

\section{Modified $\boldsymbol{J}$ doubling}

$J$ doubling in the frequency domain ${ }^{11-12}$ consists on the isolation, extraction and baseline correction of a multiplet. The signal is convolved with an array of delta functions spaced by a trial $J, J^{*}$. This method uses a set of delta functions defined by: (..., $\left.1,-1,1,-1,1,-1, \ldots\right)$, meanwhile the modified $J$ doubling employs a symmetric even set of delta functions (..., 1, -1, 1 , $1,-1,1, \ldots) . J^{*}$ is then varied within the interval where the real $J$ is expected, $J_{\text {Real. }}$. The absolute integral is obtained for each convoluted spectrum, and plotted versus $J^{*}$. This function is known as the integral function, Figure 2. The highest global minimum occurring at larger frequencies of the integral function within an interval corresponds to the real coupling constant. However, subharmonic conditions ${ }^{13}$ produce, as well, several minima which are not as deep as $J_{\text {Real }}$. This fact may confuse a person not familiarized with the method. However, the deepest minimum at a higher frequency always corresponds to the value of the coupling constants we want to measure. When complex multiplets require subsequent deconvolutions, this minimum is flanked by subharmonics due to additions and subtractions of the other coupling constants.
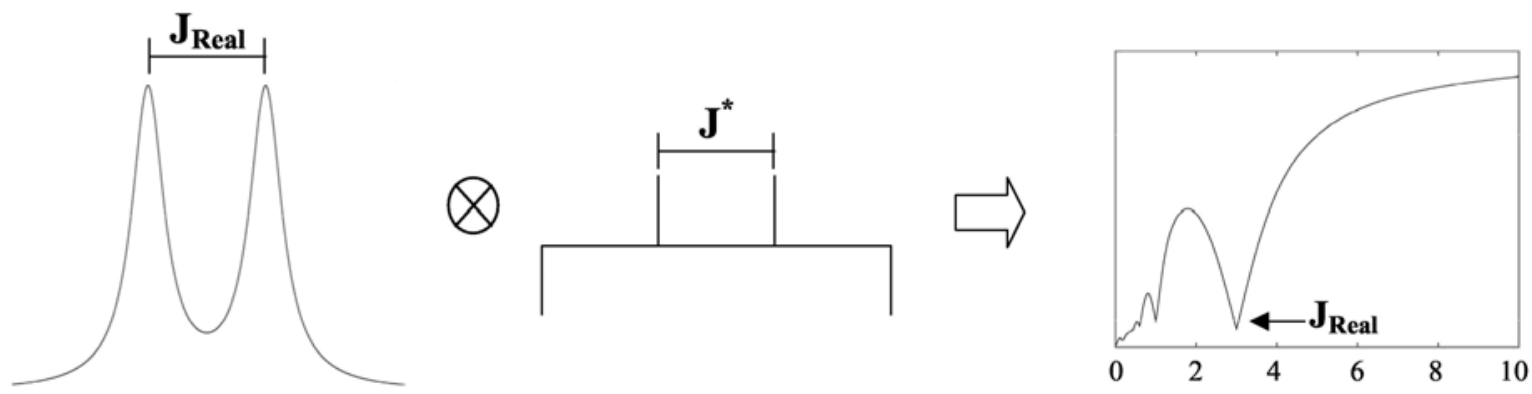

Figure 2. Scheme of modified $J$ doubling in the frequency domain.

When the $J^{*}$ matches the $J_{\text {Real }}$, the integral function has a deep local minimum. This minimum is explained in Figure 3, which is the synopsis of the modified $J$ doubling in the frequency domain when $J^{*}=J_{\text {Real }}$. We represent a doublet convoluted with a set of delta functions $(-1,1,1,-1)$ separated by $J_{\text {Real }}$. This process is represented as the sum of four steps, Figure 3b. As a first step, the doublet is multiply by -1 .In the second step, it is added to the 
doublet previously multiplied by 1 , which is moved according to the separation of the delta functions, $J_{\text {Real }}$. Thirdly, it is added again to the doublet multiplied by 1 and moved $2 J_{\text {Real }}$. Finally, it is added again to the doublet multiplied by -1 and moved $3 J_{\text {Real }}$. There are two signals that are canceled out. The result, shown in Figure 3c, corresponds to two inverted signals at the extremes and at the center the effectively deconvoluted multiplet. This method is one of the simplest that can produce the deconvoluted signal with little effort.

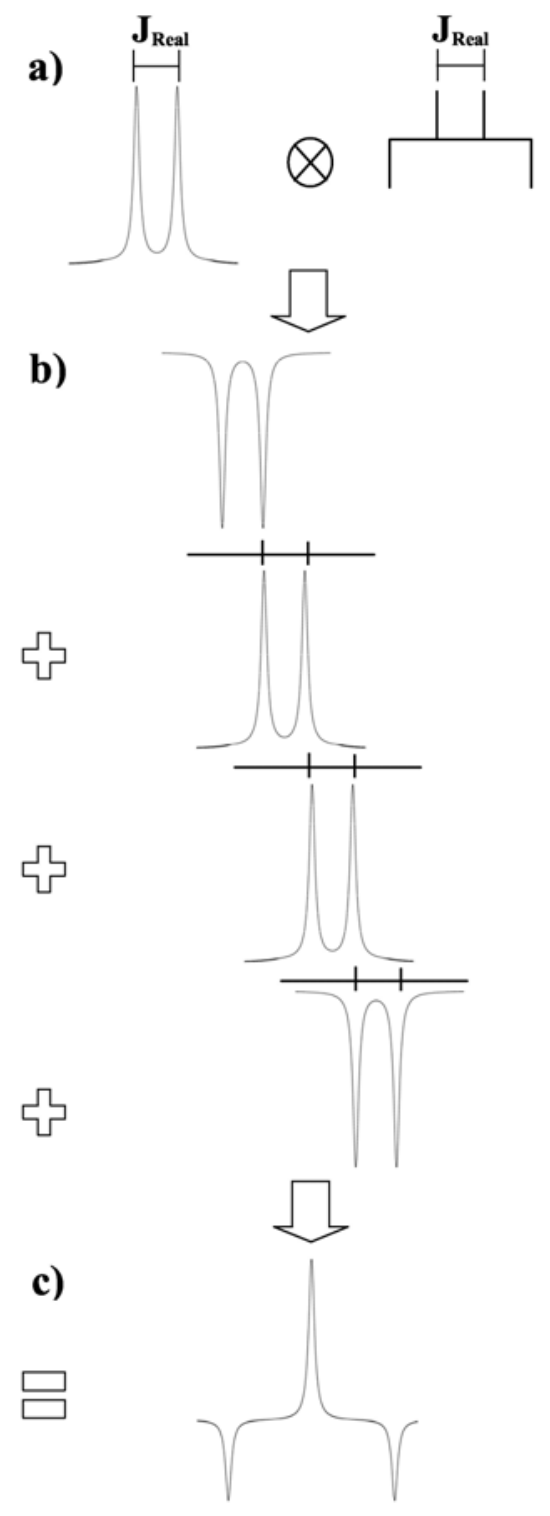

Figure 3. Synopsis of modified $J$ doubling when $J^{*}=J_{\text {Real }}$.

When $J^{*}$ are slightly different from $J_{\text {Real }}$, an increment is produced in the integral value of the generated signal. This is the reason why the minima represent the value of the coupling constant. We have tested different sets of deltas, and most of the time, they show the same integral 
function with minor variations. However, the results of using those delta functions to deconvolve a multiplet with $J_{\text {Real }}$ may vary. A great advantage of modified $J$ doubling is that it can be applied to simplify complex multiplets removing the coupling constants one by one. Modified $J$ doubling can be used step by step for pattern recognition. In the next section the main idea of this work which is the deconvolution of signals with equal $J$ 's in one single step is developed.

\section{Simultaneous deconvolution of equal couplings}

Several methods have problems when they try to measure equal o very similar coupling constants. ${ }^{3,8}$ According to our experience, $J$ doubling is not the exception; the minimum of integral functions that indicates the value of equal couplings becomes broader. In order to solve this problem, we proposed a new algorithm for simultaneous deconvolution of equal coupling constants. ${ }^{16}$ Our objective is to measure accurate coupling constants and, at the same time, deconvolve triplets, quadruplets, quintuplets, etc., i. e. multiplets that follow the structure of Pascal's triangle.

To explain this new method, we should first consider the simplest case, a triplet, Figure 4a. Using the original $\mathrm{J}$ doubling method, a triplet is deconvolved in two steps: the coupling is measured and deconvolved to obtain a doublet; and, subsequently, deconvolved again with the same value as before. Based on modified $J$ doubling, we propose that, instead of performing this two-step process, it is possible to convolute the delta functions first, Figure 4b, and in one single step deconvolute the triplet into a singlet, Figure 4c. It is compulsory to eliminate the signals that are outside the center of the convoluted multiplet, as the modified $J$ doubling does. In Table 1 , we present the convolution of two types of delta functions with four and six elements. If delta functions are convoluted once, the new delta function $v . g .(1,-2,-1,4,-1,-2.1)$ will deconvolve a triplet into a singlet in one simple step. Accordingly, when we want to measure and deconvolve a quartet, we should convolute the delta functions two times first, v. g. $(-1,3,0,-8,6.6,-8,0,3$, -1 ), and then convolute the signal. The number of convolutions needed to deconvolve a multiplet will depend on the signal multiplicity, see Table 1.

It is important to mention one advantage and disadvantage of this new method. This type of delta functions can be used to measure any kind of multiplets, the integral functions produce the same local minima at values of real $J$ 's; but, only the correct new delta function will deconvolve into the right multiplet. Unfortunately, multiple convolutions of delta functions produce really high numbers generating greater artifacts as mentioned elsewhere. ${ }^{10}$ This problem also limits the number of delta functions to be used, 16 is a recommended number; and, it restricts the method to deconvolve in one single step simulated multiplets, in the best conditions up to octuplets. Nevertheless, the general procedure is based on the modified $J$ doubling method, which seems to be the formal deconvolution process. ${ }^{13}$ This conception allows us to use the mathematical properties of the convolution theorem and the associative property in the frequency domain.

Table 1. Convolution of delta functions 


\begin{tabular}{|c|c|c|c|}
\hline Delta Function & $\begin{array}{c}\text { Number of } \\
\text { Convolutions }\end{array}$ & New Delta Function & $\begin{array}{c}\text { Deconvolved } \\
\text { Multiplet }\end{array}$ \\
\hline \multirow[t]{3}{*}[-1,1,1,-1]{} & 1 & $1,-2,-1,4,-1,-2,1$ & triplet \\
\hline & 2 & $-1,3,0,-8,6,6,-8,0,3,-1$ & quartet \\
\hline & 3 & $1,-4,2,12,-17,-8,28,-8,-17,12,2,-4,1$ & quintuplet \\
\hline \multirow[t]{2}{*}[1,-1,1,1,-1,1]{} & 1 & $1,-2,3,0,-3,6,3,0,3,-2,1$ & triplet \\
\hline & 2 & $1,-3,6,-4,-3,15,-17,9,9,-17,15,-3,-4,6,-3,1$ & quartet \\
\hline
\end{tabular}

a)

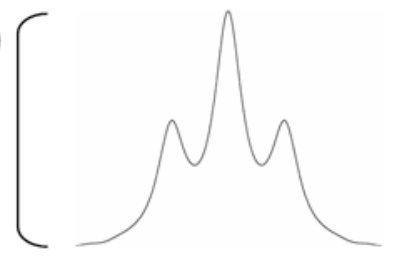

b)

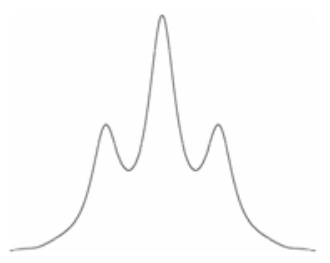

c)

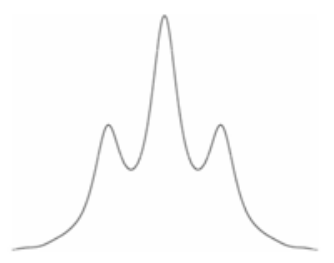

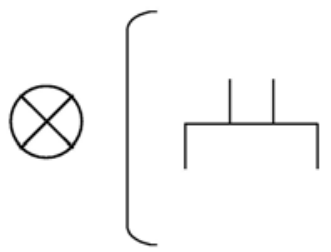

$\sqrt{5}$

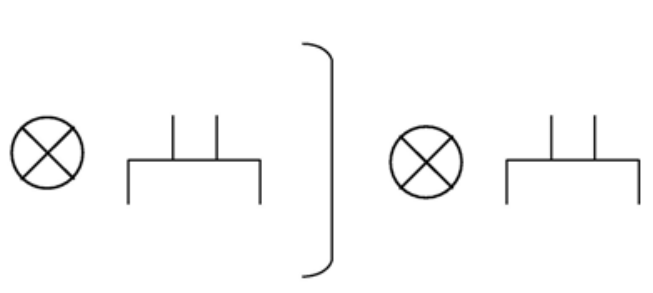

or

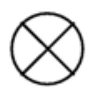

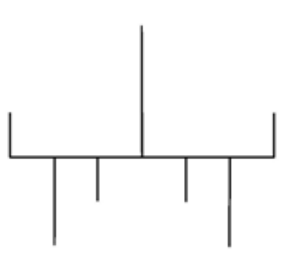

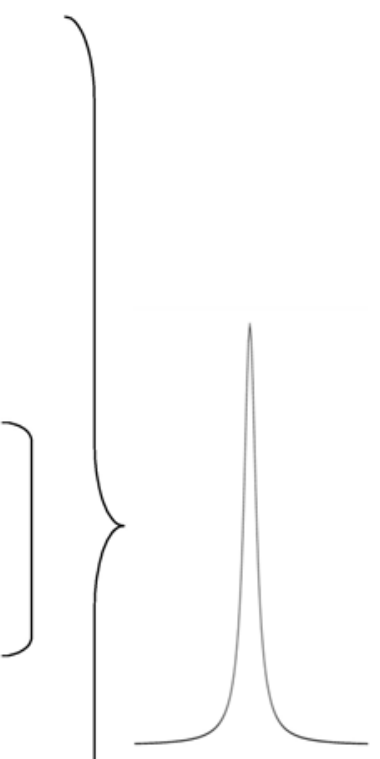

Figure 4. Schematic representation of the new algorithm for simultaneous deconvolution of equal spin-spin couplings. 


\section{Results and Discussion}

\section{Pattern recognition}

As mentioned before, pattern recognition is a deconvolution process. A very simple case is a doublet of doublets; Figure 5 represents this case, where the multiplet is defined by $J_{x}>J_{y}$. Modified $J$ doubling can be used to measure both couplings at the same time and remove one by one. In our experience, it is recommended to remove the higher coupling constants first, $J_{x}$; this process is represented in Figure $5 \mathrm{a}$ and $5 \mathrm{~b}$. The deconvolved signal is then convolved again and the smallest coupling constant is removed to generate a singlet, Figure 5e. The modified $J$ doubling process is completely linear; so, it is also possible to remove the smallest coupling constant first, $J_{y}$, Figure 5c and 5d; and, at the end, remove the bigger coupling constant, $J_{x}$. The resulting signal is independent of the procedure followed, Figure 5e.

a)

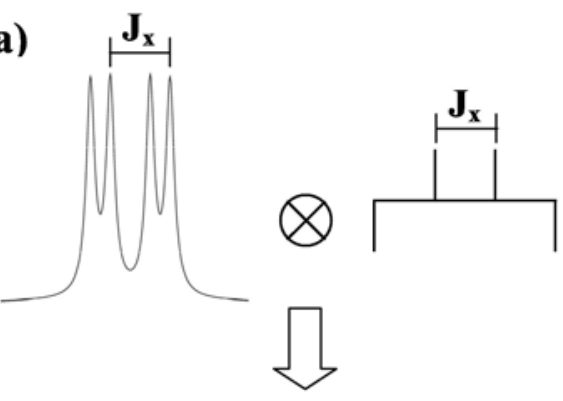

b)
$\mathbf{J}_{\mathbf{H}}$

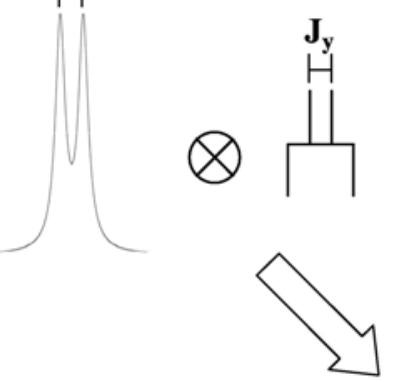

c)

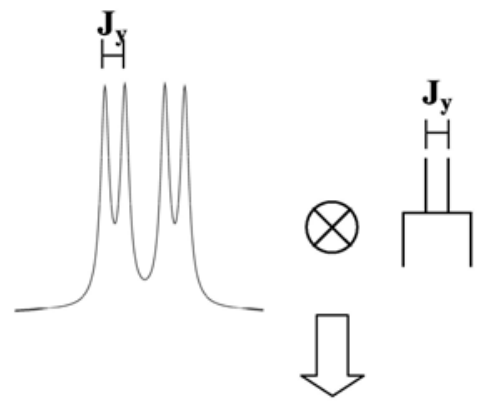

d)
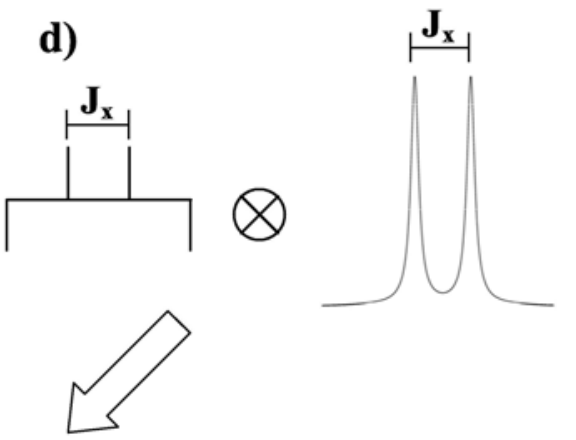

e)

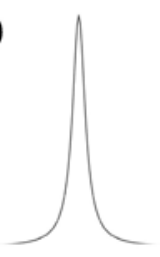

Figure 5. Two different procedures for deconvolute a simulated doublet of doublets. 
We applied the process to a very simple experimental signal, the aromatic H2' multiplet of nicotine, Figure 6a. The procedure consists on digitizing, isolating, baseline correcting and extracting the multiplet. Once these have been done, the signal is processed by the modified $J$ doubling method using 64 delta functions. The integral function, Figure 6b, clearly shows several minima at 2.24, 0.88, 0.76, $0.44 \mathrm{~Hz}$. The $2.24 \mathrm{~Hz}$ minimum corresponds to the biggest coupling constant present in the multiplet. The next two minima are too close, making it difficult to identify the appropriate coupling constant. However, the first subharmonic condition is at $J$ / 3 . Solely considering these data, it seems that the minimum at $0.88 \mathrm{~Hz}$ is another coupling constant and the minimum at 0.76 is the subharmonic of $2.24 \mathrm{~Hz}$. The first deconvoluted signal is a double of doublets, Figure 6c. The integral plot of this signal is in Figure 6d. The function presents three clear minima at $0.88,0.45$, and $0.29 \mathrm{~Hz}$. The minimum at $0.76 \mathrm{~Hz}$ of the first integral function disappears, confirming our supposition that it was a subharmonic. These results confirm that $0.88 \mathrm{~Hz}$ corresponds to another coupling constant with a subharmonic condition at $0.29 \mathrm{~Hz}$. Another coupling constant seems located around $0.45 \mathrm{~Hz}$. The deconvoluted signal without the coupling constant at $0.88 \mathrm{~Hz}$ is presented in Figure 6e. A third process produces the integral function presented in Figure 6f. At this point, it is clear that there is only one coupling constant left at $0.44 \mathrm{~Hz}$. When this coupling is removed, the signal became a singlet, Figure $6 \mathrm{~g}$. Further treatment will show that there is no other coupling constant bigger than $0.17 \mathrm{~Hz}$, Figure $6 \mathrm{~h}$. This analysis determines the coupling constant for nicotine: $J_{H 2^{\prime}, H 4^{\prime}}=2.24 \mathrm{~Hz}, J_{H 2^{\prime}, H 5^{\prime}}=0.88$ $\mathrm{Hz}$, and $J_{H 2^{\prime}, H 2}=0.44 \mathrm{~Hz}$. It is also possible to say that the coupling constant $J_{H 2^{\prime}, H 6^{\prime}}$ is equal or smaller than $0.17 \mathrm{~Hz}$, where the inflection point can be observed in Figure 6h. These values are in complete agreement with those previously reported. ${ }^{17}$

This procedure has been applied to a simulation with coupling constants of 8.70, 5.30, 3.40 and $3.40 \mathrm{~Hz}$ previously reported by Hoye, ${ }^{1}$ Figure 7 . For this signal, Figure $7 \mathrm{a}$, it is really difficult to recognize the pattern that it is producing the splitting. According to our method, firstly the signal is convoluted. The integral function, Figure 7b, clearly shows minima at 8.7, 5.3 and a broad minimum at $3.4 \mathrm{~Hz}$. As mentioned before, it is convenient to deconvolve the original signal and repeat the process again. The second integral function is plotted in Figure 7c. We can measure a coupling constant at 5.3 again and a broad minimum at $3.4 \mathrm{~Hz}$, Figure $7 \mathrm{~d}$. The $5.3 \mathrm{~Hz}$ coupling is removed, Figure 7e. At this stage, it is clear that the original multiplet in Figure 7a is a double of double of triplets and with the advantage that we have measured the couplings very accurately. We apply the simultaneous deconvolution method to measure the other two coupling constants, Figure 7f. Simultaneous deconvolution could be applied to Figure 7e to obtain a singlet in one single step, Figure $7 \mathrm{~g}$. 


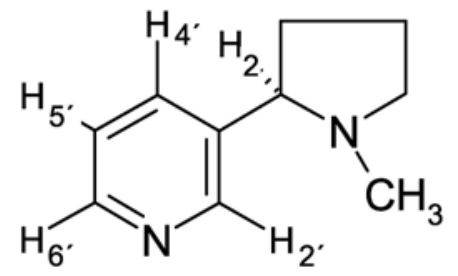

a)

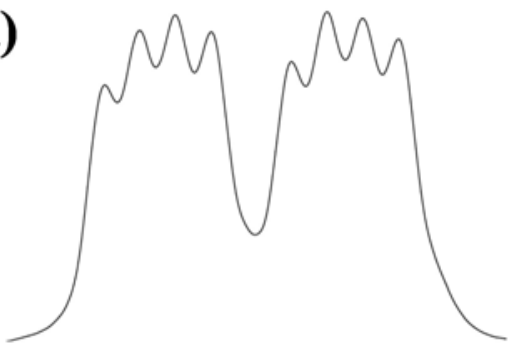

c)

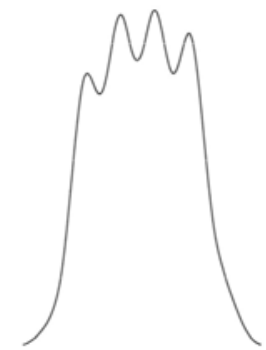

e)

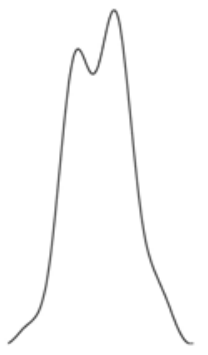

g)

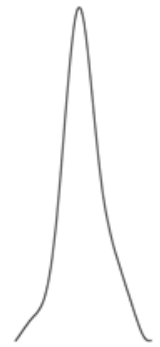

b)

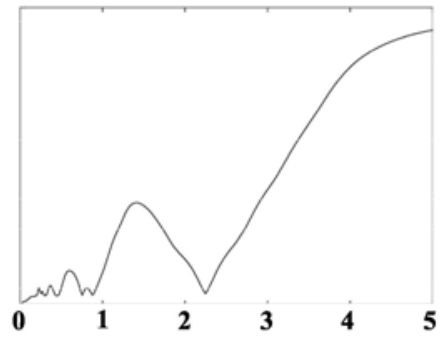

d)

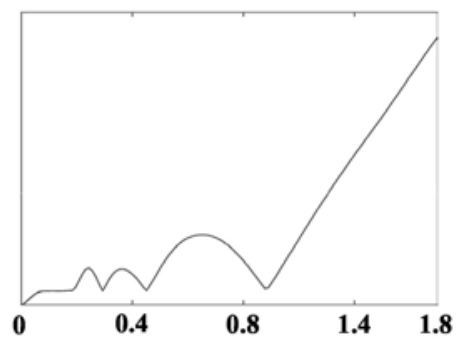

f)

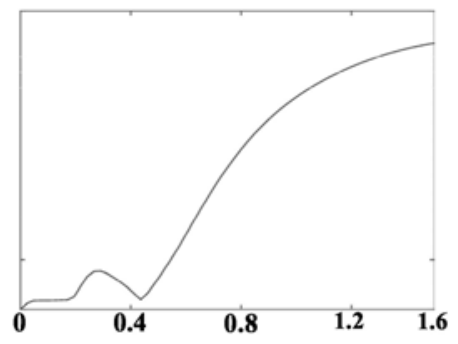

h)

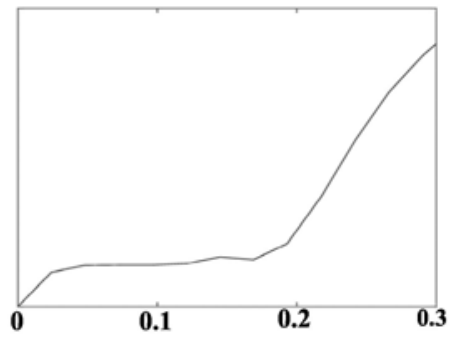

Figure 6. Complete deconvolution of the experimental signal from H2' in nicotine. 
a)

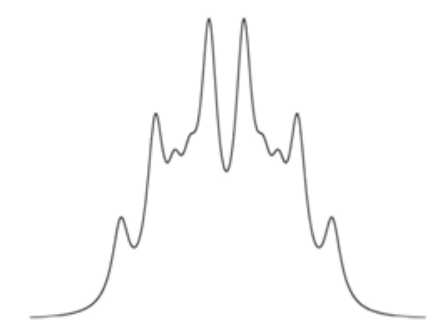

c)

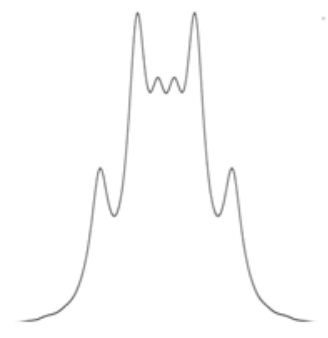

e)

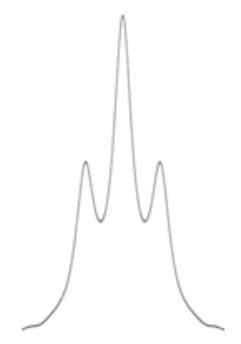

b)

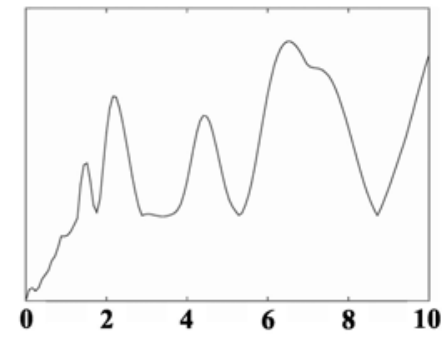

d)

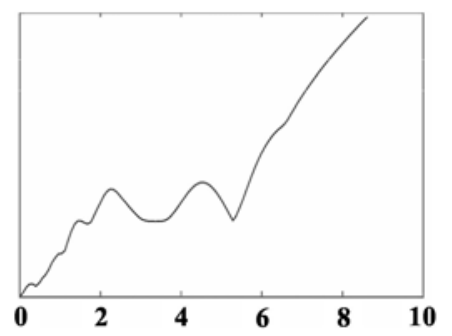

f)

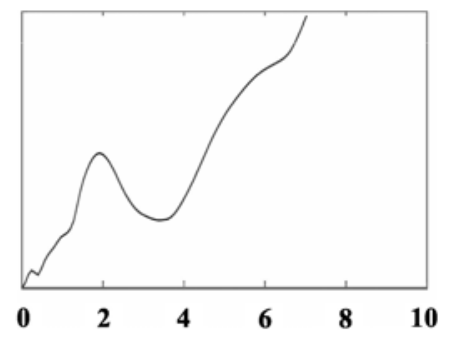

g)

Figure 7. Systematic deconvolution of a double of double of triplets, showing the integral function for each step.

We show simultaneous deconvolutions applied to a simulated triplet of quintuplets in Figure 8a. A single deconvolution removes the coupling constant at bigger frequencies, Figure 8b. A different process, taking away two couplings at the same time is shown in Figure 8c. The signal, a triplet of quintuplets, becomes a quintuplet in one single step. Also, similar independent steps can be performed with the small coupling, Figure 8d-8g. We want to emphasize that the deconvolution of four couplings in one single step transforms the triplet of quintuplets, Figure 8a, into a triplet, Figure 8g. 


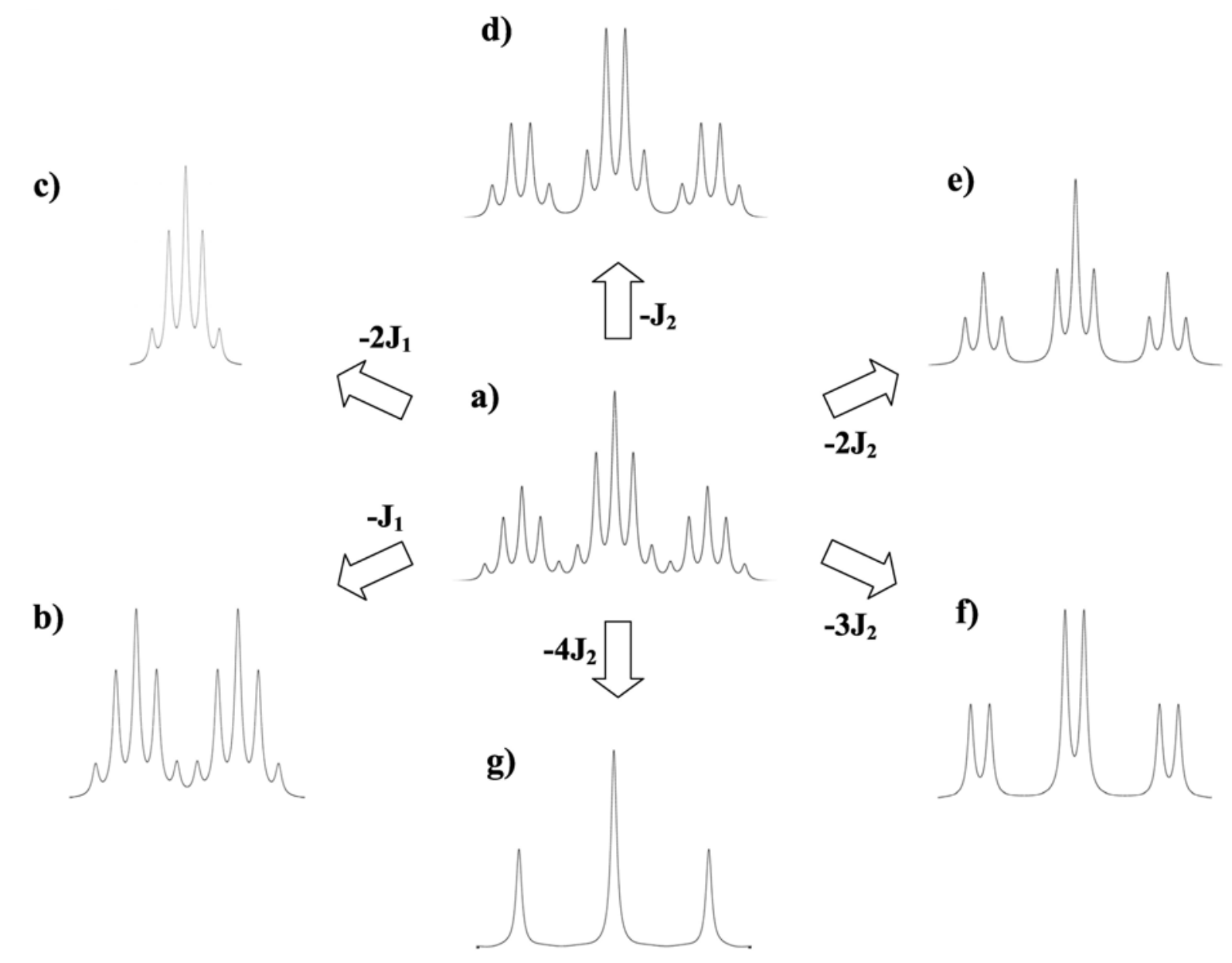

Figure 8. Different forms to deconvolute a complex multiplet in one step.

An additional advantage of simultaneous deconvolution is the possibility of measuring coupling constants within the signal. Figure 9a is the experimental proton signal of the methyl group of 1-[(4'-methyl phenyl)-azo]-2-naphtol, which shows an unusual pattern. Considering that the methyl group can only couple with ortho phenyl protons, H3'; we measured two coupling constants of $0.71 \mathrm{~Hz}$, Figure 9b, with a convolution process of 16 delta functions and deconvolved it in a single step, Figure 9c. After analyzing the deconvolved signal, a triplet line shape can be observed instead of the expected singlet, probably due to the coupling with the meta phenyl protons, H2', as well. We applied the same procedure again and found in the integral function a minimum at $0.38 \mathrm{~Hz}$, which corresponds to a small minimum, Figure 9d. We end up with a singlet after a second simultaneous deconvolution, Figure 9e. For comparison purposes, in Figure 9f we have re-plotted the experimental signal and in Figure 9g the simulated signal; it is not possible to differentiate them. 

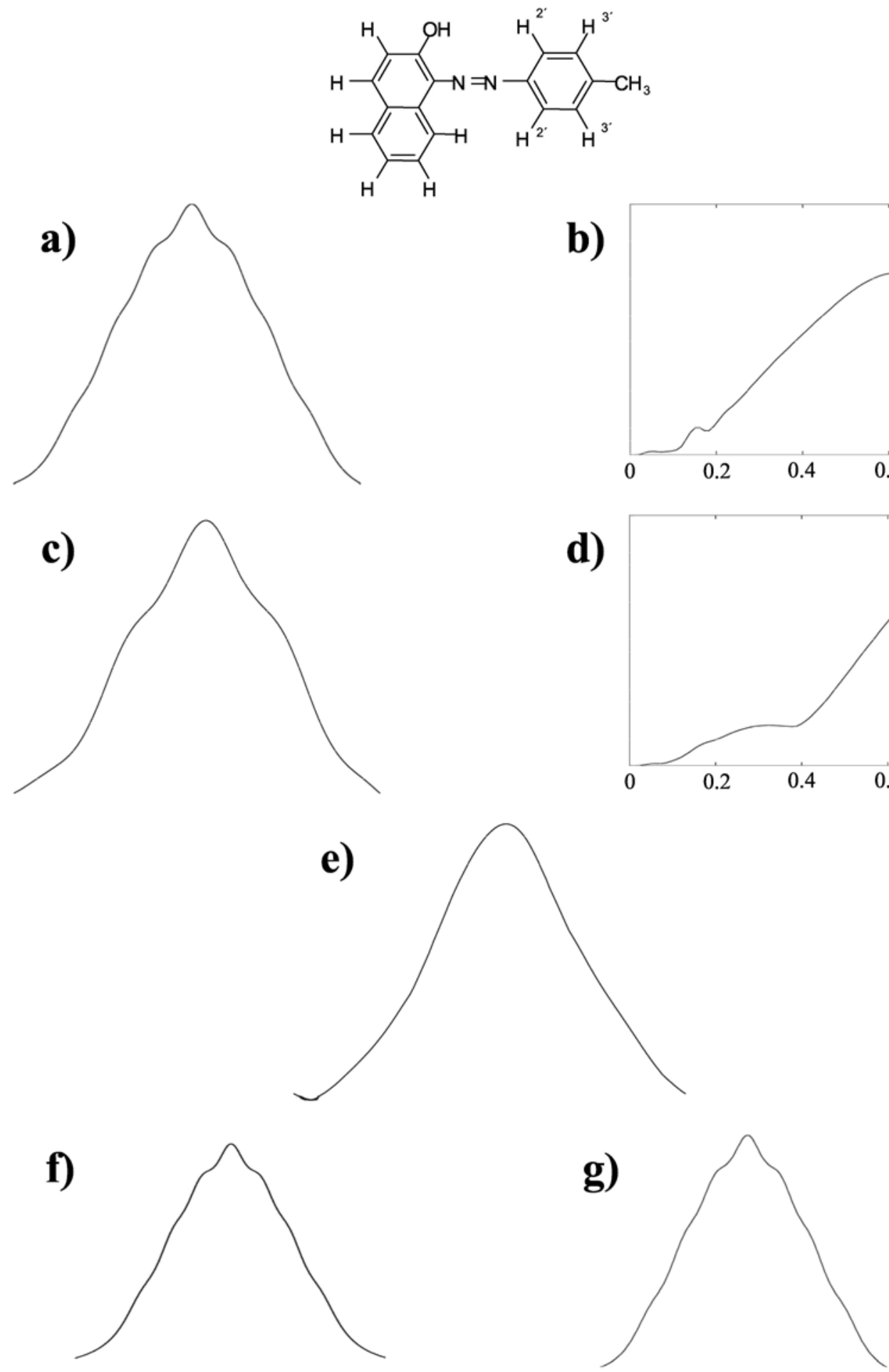

Figure 9. Experimental signal deconvolved twice with different $J$ values and comparison between the experimental data with the simulated one. 


\section{Conclusions}

We have shown that modified $J$ doubling is a simple and robust method for measuring accurately spin-spin coupling constants. At this moment, this is the only method able for measuring very small coupling constants immersed in complex multiplets and within the linewidth at the same time. This new information is not possible to achieve by simple analysis. It is important to stress that this procedure is based on the mathematical properties of the convolution theorem and uses the associative property in the frequency domain. Furthermore, using only one single step in the deconvolution process reduces the computational effort and provides direct information on the multiplet nature, even for non-common patterns.

\section{Experimental Section}

\section{NMR simulations}

All NMR signals simulations were done using our program with a digital resolution of $0.01 \mathrm{~Hz}$ per point and noise free. Most single step calculations were done using 32 delta functions. For simultaneous deconvolution, 16 delta functions were convoluted the number requested by the multiplets, one for triplets, two for quartets, and so on.

\section{NMR experiments}

A sample of 1-[(4'-methyl phenyl)-azo]-2-naphtol purified by re-crystallization, $10 \mathrm{mg}$ where solved in $0.7 \mathrm{ml}$ of CDCl3. Nicotine was used as it is from Aldrich, and $10 \mathrm{mg}$ where solved in $0.7 \mathrm{ml}$ of $\mathrm{CDCl}_{3}$. NMR ${ }^{1} \mathrm{H}$ experiment used 8 seconds of acquisition time and Fourier transformed without apodization using zero filling enough to obtain a digital resolution better than $0.02 \mathrm{~Hz}$ per point.

\section{Acknowledgments}

This work was partially supported by CONACYT grant 38616E. ESM and VCC gratefully acknowledge scholarships by CONACYT. The authors would like to acknowledge Gabriela Salcedo for language correction. Source C program for fast deconvolution is available under request to the authors. 


\section{References}

1. Hoye, T. R.; Hanson, P. R.; Vyvyan, J. R. J. Org. Chem. 1994, 59, 4096.

2. Hoye, T. R.; Zhao, H. J. Org. Chem. 2002, 67, 4014.

3. Golotvin, S.; Vodopianov, E.; Williams, A. Magn. Reson. Chem. 2002, 40, 331.

4. Eberstadt, M.; Gemmecker, G.; Mierke, D. F.; Kessler H. Angew. Chem. Int. Ed. Engl. 1995, $34,1671$.

5. Le Parco, J. M.; McIntyre, L.; Freeman, R. J. Magn. Reson. 1992, 97, 553.

6. McIntyre, L.; Freeman, R. J. Magn. Reson. 1992, 96, 425.

7. Freeman, R.; McIntyre L. Israel J. Chem. 1992, 32, 231.

8. Stonehouse, J.; Keeler, J.; J. Magn. Reson. 1995, A112, 43.

9. Huber, P.; Bodenhausen, G. J. Magn. Reson. 1993, A102, 81.

10. Jeannerat, D.; Bodenhausen, G. J. Magn. Reson. 1999, 141, 133.

11. Blechta, V.; del Río-Portilla, F.; Freeman, R. Magn. Reson. Chem. 1994, 32, 134.

12. del Río-Portilla, F.; Blechta, V.; Freeman, R. J. Magn. Reson. 1994, A111, 132.

13. Garza-García, A.; Ponzanelli-Velázquez, G.; del Río-Portilla, F. J. Magn. Reson. 2001, 148, 214.

14. A delta function is a relation which is zero in the entire domain except in specific points, where usually the value is \pm 1 .

15. Mahi, L.; Duplan, J. C. J. Magn. Reson. 2003, 162, 341.

16. Presented for the first time in the $44^{\text {th }}$. Experimental Nuclear Magnetic Resonance Conference, Savannah, Georgia, U. S. A. April, 2003.

17. del Río-Portilla, F.; Freeman, R. J. Magn. Reson. 1994, A108, 124. 